\title{
Group Consensus for Discrete-Time Heterogeneous Multiagent Systems with Input and Communication Delays
}

\author{
Yiliu Jiang, ${ }^{1,2}$ Lianghao Ji (iD, ${ }^{1,2}$ Xingcheng Pu ${ }^{(D)}{ }^{3}$ and Qun Liu ${ }^{1,2}$ \\ ${ }^{1}$ Chongqing Key Laboratory of Computational Intelligence, Chongqing University of Posts and Telecommunications, \\ Chongqing 400065, China \\ ${ }^{2}$ School of Computer Science and Technology, Chongqing University of Posts and Telecommunications, Chongqing 400065, China \\ ${ }^{3}$ School of Science, Chongqing University of Posts and Telecommunications, Chongqing 400065, China
}

Correspondence should be addressed to Lianghao Ji; lianghao.ji@gmail.com

Received 11 January 2018; Revised 24 August 2018; Accepted 26 September 2018; Published 17 October 2018

Academic Editor: Marcio Eisencraft

Copyright (C) 2018 Yiliu Jiang et al. This is an open access article distributed under the Creative Commons Attribution License, which permits unrestricted use, distribution, and reproduction in any medium, provided the original work is properly cited.

Group consensus seeking is investigated for a class of discrete-time heterogeneous multiagent systems composed of first-order and second-order agents with both communication and input time delays. Considering two types of system topologies, novel protocols based on the competitive and cooperative relationships among the agents are presented, respectively. By matrix theory and frequency domain analysis method, the sufficient conditions solving consensus problem are obtained. The results show that the achievement of group consensus is bound up with the input time delays, coupling weights between the agents and the system's control parameters, but it is irrelevant to the communication delays. Finally, numerical simulations are presented to illustrate the correctness of the theoretical results.

\section{Introduction}

Multiagent systems (MASs) have recently attracted widespread concern. It not only has extensive application in distributed sensor networks, formation control, and artificial intelligence, but also contains great potential value for homeland security and military affairs. Consensus, a typical and crucial issue of the coordination of MASs, refers to that all the agents asymptotically achieve certain global agreement only via the information interactions between them. As an extended problem of consensus, group consensus means that different consensus states can be reached by the different subgroups of a complex system while there is no consensus between those subgroups. It has been widely used to handle multitasking problems and the decomposition of large-scale tasks. So far, there has been a lot of research on consensus and group consensus of MASs, such as [1-5].

Note that most of the existing work is established mainly on homogeneous agents, given that all agents in one complex system possess identical dynamics. However, the difference of the agent dynamics is ubiquitous in practical applications, which is the result of external impacts or interaction restrictions of system. Furthermore, we usually have the demand of using multiple agents with different dynamics to reduce control costs. Thus, the consensus problem of heterogeneous MASs is increasingly being studied, which is also a more complex and challenging task, such as [6], where certain conditions were presented to guarantee the consensus of heterogeneous MASs under fixed and switching topology. In [7], Sun considers the consensus tracking control problem for general linear multiagent systems with unknown dynamics. Jiang [8] investigated the consensus of the heterogeneous networks with the influence of time delays. In [9], for discrete-time heterogeneous systems, Sun discussed both the sufficient conditions to ensure consensus and the consensus equilibrium point based on the graph theory and the matrix theory. Liu [10] proposed some sufficient conditions for heterogeneous multiagent systems to achieve consensus with sampled bounded communication delays. In [11], Bernardo et al. discussed the consensus of time-varying heterogeneous communication delays and applied this strategy for platooning of vehicles. 
At the same time, due to the application demands of multitasking, the group consensus problem of MASs is now occupying research hotspots [12-14]. Currently, methods for achieving group consensus include common cooperationbased strategies [15-19] and fewer competition-based strategies [20]. Since the analysis of the group consensus problem of heterogeneous systems is a more complex work than that of homogeneous system, there are few related articles. This incites us to study the group consensus problem for heterogeneous multiagent systems. The main contributions of this paper are as follows. First, a heterogeneous system is established which is composed of agents with first-order and second-order dynamics. And the sufficient algebraic conditions are correspondingly deduced to ensure the consensus of the system. The dynamics of the first-order agents in our system model does not include the virtual velocity, which exists in $[15,17,19]$ but reduces the flexibility of the system. Second, utilizing the characteristics of two types of common topologies, effective control algorithms to achieve group consensus are proposed based on competitive and cooperative relationships, respectively. Finally, note that there exists communication delay when the channel is congested and the input delay when the sensor is aging or the computing power is insufficient, while most of articles like [10, 15, 18] did not consider any or just consider one of them. The two kinds of delays are reasonably considered in the agents' dynamics of our system. Utilizing frequency domain analysis and Gerschgorin circle theorem, we derived the upper bound of input time delay to guarantee the consensus of system. Meanwhile, discrete-time based systems are common in the real world, and current related research is focused on continuous systems [10-19]. Hence, it is also of practical values to investigate the consensus problem of the discrete-time systems. The above innovations make our system model closer to reality and having a wider scope of application.

The remaining of this paper is organized as follows. In Section 2, we first list some preliminaries about graph theory, lemmas, and problem statements. Section 3 contains the main analysis of the group consensus of discrete-time heterogeneous MASs with multiple time delays. In Section 4, several simulations are demonstrated to validate the theoretical results. Finally, some conclusions are drawn based on our work.

Note. Throughout this paper, we use $\mathbb{R}, \mathbb{R}^{n}, \mathbb{R}^{n \times m}$ denoting the one-dimensional Euclidean space, the $\mathrm{n}$-dimensional real vector space, and the $n \times m$ dimensional real matrices, respectively; $I_{N}$ represents the identity matrix with $N$-dimension; $\mathbb{C}$ indicates complex numbers sets; the modulus and the real parts of $\forall z \in \mathbb{C}$ are represented as $|z|$ and $\operatorname{Re}(z)$; $\operatorname{det}(\Lambda)$ and $\lambda_{i}(\Lambda)$ indicate the determinant and the $i$ th eigenvalue of matrix $\Lambda$.

\section{Preliminaries and Problems Statements}

To assist our work, some definitions, lemmas, and statements are first introduced.
2.1. Graph Theory and Interconnection Topology. Based on graph theory, in a MAS with $n$ agents, the agents and the information exchange between them can be described by a weighted directed graph (digraph) $G=(V, E, A)$, where $V=$ $\left\{v_{1}, v_{2}, \ldots, v_{n}\right\}$ denotes the node set, $E=V \times V$ denotes the edge set, and $A=\left(a_{i j}\right)_{n \times n} \in \mathbb{R}^{n \times n}$ denotes the adjacency matrix. $e_{i j}=(i, j) \in E$ denotes that there exists a directed edge from node $j$ to node $i$ in the digraph $G$ so that node $i$ can receive information from node $j . a_{i j} \neq 0$ if $e_{i j} \in E$; in addition, we assume $a_{i i}=0$ for all $i \in 1, \ldots, n$.

Meanwhile, the neighbor set and the in-degree of $i$ th node are represented as $N_{i}=\left\{j \in V: e_{i j} \in E\right\}$ and $d_{i}=$ $\operatorname{deg}_{\text {in }}(i)=\sum_{j \in N_{i}} a_{i j}$, respectively. Denote in-degree matrix as $D=\operatorname{diag}\left\{d_{1}, d_{2}, \ldots, d_{n}\right\}$, then Laplacian matrix $L=D-A$.

A directed spanning tree consists of nodes with one and only one directed edge for receiving information from other nodes more than a node called the root. A graph which has a directed spanning tree means that there exists such a tree that is composed of several edges and all the nodes of this graph.

Definition 1. There is a graph $G=(V, E)$ which can be called bipartite graph when the following two conditions are satisfied:

(i) The vertex $V$ can be completely divided into two disjoint subsets $V_{1}$ and $V_{2}$.

(ii) The two vertexes $i, j$ associated with each edge $e=$ $(i, j)$ of the graph belong to different subset $i \in V_{1}$, $j \in V_{2}$.

Lemma 2 (see [21]). Consider a complex network with bipartite digraph topology which has a directed spanning tree, it then has $\operatorname{Re}\left(\lambda_{i}(D+A)\right)>0$ when $\lambda_{i}(D+A) \neq 0$ and $\operatorname{rank}(D+A)=$ $n-1$.

Lemma 3 (see [20]). Suppose that $z=\left[z_{1}, z_{2}, \ldots, z_{n}\right]$ and $L \in$ $\mathbb{R}^{n \times n}$ is a Laplacian matrix. Then, the following four conditions are equivalent:

(i) $\lambda_{i}(L), i \in n$ all have positive real parts except a simple zero eigenvalue;

(ii) $L z=0$ indicates that $z_{1}=z_{2}=\cdots=z_{n}$;

(iii) the consensus is asymptotically achieved for a system $z=-L z$;

(iv) the directed graph represented by $L$ has at least one directed spanning tree.

2.2. Discrete-Time Heterogeneous Multiagent System. Consider a discrete-time heterogeneous MASs including $n+$ $m$ agents. For the purposes of discussion, we assume the second-order agents are $1, \ldots, n$ and the first-order agents are represented by the remaining $n+1, \ldots, n+m$. Hence, the dynamics of the system are as follows:

$$
\begin{aligned}
& x_{i}(k+1)=x_{i}(k)+v_{i}(k), \\
& v_{i}(k+1)=v_{i}(k)+u_{i}(k),
\end{aligned}
$$




$$
x_{l}(k+1)=x_{l}(k)+u_{l}(k), \quad l \in \sigma_{2},
$$

where $\sigma_{1}=\{1,2, \ldots, n\}, \sigma_{2}=\{n+1, n+2, \ldots, n+m\}, \sigma=\sigma_{1} \cup$ $\sigma_{2}$, using $u_{i}(k), x_{i}(k), v_{i}(k) \in \mathbb{R}$ to represent control input, position state, and velocity state of $i$ th agent, respectively.

For heterogeneous MASs, each agent $i$ may have secondorder and first-order neighbors, which can be described as $N_{i, s}$ and $N_{i, f}$ separately. Accordingly, all the neighbors of $i$ are denoted as $N_{i}=N_{i, f} \cup N_{i, s}$. Based on the different orders of the dynamics, we partition the adjacency matrix of the system $A$ as $A=\left(\begin{array}{cc}A_{s} & A_{s f} \\ A_{f s} & A_{f}\end{array}\right)$, where $A_{s} \in \mathbb{R}^{n \times n}$ and $A_{f} \in \mathbb{R}^{m \times m}$ denote adjacency matrix consisting only of second-order and first-order nodes, $A_{s f}$ is composed of the adjacency weights among the nodes that are from the secondorder to first-order, and $A_{f s}$ indicate the opposite meaning. Meanwhile, we rewritten the Laplacian matrix $L$ as $L=D$ $A=\left(\begin{array}{cc}L_{s}+D_{s f} & -A_{s f} \\ -A_{f s} & L_{f}+D_{f s}\end{array}\right)$, where $L_{s}$ and $L_{f}$ indicate the Laplacian matrix considering second-order and first-order nodes with their connections, $D_{s f}=\operatorname{diag}\left\{\sum_{j \in N_{i, f}} a_{i j}, i \in \sigma_{1}\right\}$, and $D_{f s}=$ $\operatorname{diag}\left\{\sum_{j \in N_{i, s}} a_{i j}, i \in \sigma_{2}\right\}$. Thus, we can rewrite matrix $D+A$ as $D+A=\left(\begin{array}{cc}D_{s}+A_{s}+D_{s f} & A_{s f} \\ A_{f s} & D_{f}+A_{f}+D_{f s}\end{array}\right)$.

Definition 4. The discrete-time heterogeneous MAS (1) and (2) can asymptotically reach consensus if and only if the following two conditions hold for any initial states:

(i) $\lim _{k \rightarrow \infty}\left\|x_{i}(k)-x_{j}(k)\right\|=0$, for $i, j \in \sigma_{k}(k=1,2)$;

(ii) $\lim _{k \rightarrow \infty}\left\|v_{i}(k)-v_{j}(k)\right\|=0$, for $i, j \in \sigma_{1}$.

Lemma 5 (see [22]). The inequality $\sin (((2 D+1) / 2) \omega) /$ $\sin (\omega / 2) \leq 2 D+1, \omega \in[-\pi, \pi]$ holds for all nonnegative integers $D$.

\section{Main Results}

Next, we will investigate the group consensus problem for the discrete-time heterogeneous MASs with communication and input time delays under two typical topologies.

\subsection{Couple-Group Consensus for Discrete-Time Heterogeneous} MASs with Multiple Time Delays under Bipartite Digraph. Most of the existing works, such as [15-19], are based on the agents' cooperative relationship. From a different point of view, a novel group consensus protocol was proposed in [9] referring the characteristic of the bipartite digraph, which is based on the competitive relationship between the agents. Inspired by their work, two novel delayed protocols based on the competitive relationship are designed, which are listed as follows:

$$
\begin{aligned}
u_{i}(k)= & -\alpha\left[\sum_{j \in N_{i}} a_{i j}\left[x_{j}\left(k-\tau_{i j}\right)+x_{i}(k-\tau)\right]\right] \\
& -\beta v_{i}(k-\tau), \quad i \in \sigma_{1}
\end{aligned}
$$

and

$$
\begin{aligned}
& u_{l}(k)=-\gamma\left[\sum_{j \in N_{l}} a_{l j}\left[x_{j}\left(k-\tau_{l j}\right)+x_{l}(k-\tau)\right]\right], \\
& l \in \sigma_{2},
\end{aligned}
$$

where $\tau_{i j}$ denotes the communication time delays from the agent $j$ to agent $i$ and $\tau$ denotes the input time delays. $\alpha, \beta, \gamma>$ 0 are the control parameters of the systems. And we assume $a_{i j}>0$ if there exists an interaction among nodes $i$ and $j$. The closed form of the heterogeneous systems (1) and (2) with the protocols (3) and (4) is shown as

$$
\begin{aligned}
x_{i}(k+1)= & x_{i}(k)+v_{i}(k), \\
v_{i}(k+1)= & v_{i}(k) \\
& -\alpha\left[\sum_{j \in N_{i}} a_{i j}\left(x_{j}\left(k-\tau_{i j}\right)+x_{i}(k-\tau)\right)\right] \\
& -\beta v_{i}(k-\tau), \quad i \in \sigma_{1},
\end{aligned}
$$

and

$$
\begin{array}{r}
x_{l}(k+1)=x_{l}(k) \\
-\gamma\left[\sum_{j \in N_{l}} a_{l j}\left(x_{j}\left(k-\tau_{l j}\right)+x_{l}(k-\tau)\right)\right], \\
\quad l \in \sigma_{2} .
\end{array}
$$

Theorem 6. Couple-group consensus for heterogeneous MASs (5) and (6) under bipartite digraph topology with a spanning tree can be achieved asymptotically if the following conditions are satisfied:

(i) There exists a real number $\omega_{i 0} \geq 0$ satisfying the equation $\alpha d_{i}=4 \sin \left(\omega_{i 0} / 2\right) \cos \left((1+2 \tau) \omega_{i 0} / 2\right) \tan \left(\omega_{i 0} / 2\right)$ so that the inequality $2 \beta-\alpha d_{i}>4 \sin \left(\omega_{i 0} / 2\right) \sin ((1+$ $\left.2 \tau) \omega_{i 0} / 2\right)$ holds;

(ii) $\beta^{2} / \alpha(\beta+2)>\max \left\{d_{i}\right\}, \gamma<1 / \max \left\{\widehat{d}_{i}\right\}, \tau \in[0$, $\left.\min \left\{1 / 2 \beta-1 / 2,1 / 2 \gamma \max \left\{\widehat{d}_{i}\right\}-1 / 2\right\}\right)$, where $d_{i}=$ $\sum_{j \in N_{i}} a_{i j}, i \in \sigma_{1}$ and $\widehat{d}_{i}=\sum_{j \in N_{i}} a_{i j}, i \in \sigma_{2}$.

Proof. Let (5) and (6) do $z$-transformation. Assuming $x_{i}(0)=$ 0 , it yields that

$$
\begin{aligned}
z x_{i}(z)= & x_{i}(z)+v_{i}(z), \\
z v_{i}(z)= & v_{i}(z) \\
& -\alpha\left[\sum_{v_{j} \in N_{i}} a_{i j}\left(x_{j}(z) z^{-\tau_{i j}}+x_{i}(z) z^{-\tau}\right)\right] \\
& -\beta v_{i}(z) z^{-\tau}, \quad i \in \sigma_{1}
\end{aligned}
$$


$z x_{l}(z)=x_{l}(z)$

$$
-\gamma\left[\sum_{v_{i} \in N_{l}} a_{l j}\left(x_{j}(z) z^{-\tau_{l j}}+x_{l}(z) z^{-\tau}\right)\right],
$$

where $x_{i}(z)$ and $v_{i}(z)$ represent $x_{i}(k)$ and $v_{i}(k)$ after $z$ transformation.

Rewriting (7) to vector form, let

$$
\begin{aligned}
& x_{s}(z)=\left(x_{1}(z), \ldots, x_{n}(z)\right)^{T}, \\
& x_{f}(z)=\left(x_{n+1}(z), \ldots, x_{n+m}(z)\right)^{T}, \\
& \widehat{D}+\widehat{A}= \begin{cases}z^{-\tau_{i j}} a_{i j}, & i \neq j \\
\sum_{j \in N_{i}} a_{i j} z^{-\tau}, & i=j .\end{cases}
\end{aligned}
$$

Based on the equivalent representation mentioned above, we can rewrite matrix $\widehat{D}+\widehat{A}=\left(\begin{array}{cc}\widehat{D}_{s}+\widehat{A}_{s}+\widehat{D}_{s f} & \widehat{A}_{s f} \\ \widehat{A}_{f s} & \widehat{D}_{f}+\widehat{A}_{f}+\widehat{D}_{f s}\end{array}\right)$. Then we get

$$
\begin{aligned}
(z-1)^{2} x_{s}(z)= & -\alpha\left(\widehat{D}_{s}+\widehat{A}_{s}+\widehat{D}_{s f}\right) x_{s}(z) \\
& -\alpha \widehat{A}_{s f} x_{f}(z) \\
& -(z-1) \beta e^{-\tau} x_{s}(z), \\
(z-1) x_{f}(z)= & -\gamma \widehat{A}_{f_{s}} x_{s}(z) \\
& -\gamma\left(\widehat{D}_{f}+\widehat{A}_{f}+\widehat{D}_{f_{s}}\right) x_{f}(z) .
\end{aligned}
$$

Define $x(z)=\left(x_{s}(z), x_{f}(z)\right)^{T}$, then (9) can be rewritten as

$$
(z-1) x(z)=\widehat{\Phi}(z) x(z),
$$

where

$\widehat{\Phi}(z)$

$$
=\left(\begin{array}{cc}
\frac{-(z-1)^{2} I-\alpha\left(\widehat{D}_{s}+\widehat{A}_{s}+\widehat{D}_{s f}\right)}{\beta z^{-\tau}} & \frac{-\alpha \widehat{A}_{s f}}{\beta z^{-\tau}} \\
-\gamma \widehat{A}_{f s} & -\gamma\left(\widehat{D}_{f}+\widehat{A}_{f}+\widehat{D}_{f s}\right)
\end{array}\right) .
$$

The characteristic equation of system (10) is given by

$$
\operatorname{det}((z-1) I-\widehat{\Phi}(z))=0
$$

According to Lyapunov stability theory we know that if the roots of (12) are either at $z=1$ or in the unit circle of the complex plane, the group consensus of the system can be realized. Next, we discuss these two cases separately.

When $z=1, \operatorname{det}((z-1) I-\widehat{\Phi}(z))=(\alpha / \beta)^{n}(-\gamma)^{m} \operatorname{det}(\widehat{D}+$ $\widehat{A})$. Based on Lemma 2, we know that zero is a simple eigenvalue of $\widehat{D}+\widehat{A}$. Then the roots of (12) is at $z=1$.
When $z \neq 1$, define $\operatorname{det}((z-1) I-\widehat{\Phi}(z))=\operatorname{det}(I+G(z))$, where $G(z)=-\widehat{\Phi}(z) /(z-1)$. It follows that

$$
\begin{aligned}
& G(z)=\frac{-\widehat{\Phi}(z)}{z-1} \\
& =\left(\begin{array}{cc}
\frac{(z-1)^{2} I+\alpha\left(\widehat{D}_{s}+\widehat{A}_{s}+\widehat{D}_{s f}\right)}{(z-1) \beta e^{-\tau}} & \frac{\alpha \widehat{A}_{s f}}{(z-1) \beta z^{-\tau}} \\
\frac{\gamma \widehat{A}_{f s}}{z-1} & \frac{\gamma\left(\widehat{D}_{f}+\widehat{A}_{f}+\widehat{D}_{f s}\right)}{z-1}
\end{array}\right),
\end{aligned}
$$

where $z=e^{j w}$. Based on general Nyquist criteria, one can know that the roots of (12) can be located in the unit circle of the complex plane if and only if the point $(-1, j 0)$ is not enclosed by the Nyquist curve of $G\left(e^{j w}\right)$.

According to Gerschgorin circle theorem, an area containing all the eigenvalues of the matrix (the union of a set of circles $\left.G_{i}\right)$ is described as $\lambda\left(G\left(e^{j \omega}\right)\right) \in\left\{G_{i}, i \in \sigma_{1}\right\} \cup\left\{G_{i}, \quad i \in\right.$ $\left.\sigma_{2}\right\}$. Next, we separately discuss the two cases of the secondorder $\left(i \in \sigma_{1}\right)$ and the first-order $\left(i \in \sigma_{2}\right)$ nodes.

When $i \in \sigma_{1}$, the circles are identified as

$$
\begin{aligned}
& G_{i} \\
& =\left\{x: x \in C,\left|x-\frac{\alpha}{\left(e^{j \omega}-1\right) \beta} \sum_{j \in N_{i}} a_{i j}-\frac{e^{j \omega}-1}{\beta} e^{j \omega \tau}\right|\right. \\
& \left.\leq \sum_{j \in N_{i}}\left|\frac{\alpha a_{i j}}{\left(e^{j \omega}-1\right) \beta} e^{-j \omega\left(\tau_{i j}-\tau\right)}\right|\right\} .
\end{aligned}
$$

Define $d_{i}=\sum_{j \in N_{i}} a_{i j}, i \in \sigma_{1}$ and

$$
D_{i}(\omega)=\frac{\alpha}{\left(e^{j \omega}-1\right) \beta} \sum_{j \in N_{i}} a_{i j}+\frac{e^{j \omega}-1}{\beta} e^{j \omega \tau}
$$

$D_{i}(\omega)$ denotes the center of the $\operatorname{disc} G_{i}$. Suppose the first cross of the curve $D_{i}(\omega)$ on the real axis locates at $\omega_{i 0}$. According to (15), the following equation is established

$$
\alpha d_{i}=4 \sin \frac{\omega_{i 0}}{2} \cos \frac{(1+2 \tau) \omega_{i 0}}{2} \tan \frac{\omega_{i 0}}{2} .
$$

Since the point $(-a, j 0), a \geq 1$ cannot be enclosed in $G_{i}, i \in \sigma_{1}$, we obtain

$$
\begin{gathered}
\left|-a-\frac{\alpha d_{i}}{\left(e^{j \omega_{i 0}}-1\right) \beta}-\frac{e^{j \omega_{i 0}}-1}{\beta} e^{j \omega_{i 0} \tau}\right| \\
>\sum_{j \in N_{i}}\left|\frac{\alpha a_{i j}}{\left(e^{j \omega_{i 0}}-1\right) \beta} e^{-j \omega_{i 0}\left(\tau_{i j}-\tau\right)}\right| .
\end{gathered}
$$

Based on Euler's formula and from (17), we have 


$$
\begin{aligned}
& \left|-a+\frac{\alpha d_{i}}{2 \beta}-\frac{1}{\beta}\left(\cos \omega_{i 0}(1+\tau)-\cos \omega_{i 0} \tau\right)+j\left(-\frac{\alpha d_{i}}{2 \beta} \cdot \frac{\sin \omega_{i 0}}{1-\cos \omega_{i 0}}+\frac{1}{\beta}\left(\sin \omega_{i 0}(1+\tau)-\sin \omega_{i 0} \tau\right)\right)\right| \\
& \quad>\sum_{j \in N_{i}}\left|\frac{\alpha a_{i j}}{\beta}\left\{-\frac{1}{2} \cos \omega_{i 0}\left(\tau-\tau_{i j}\right)-\frac{\sin \omega_{i 0} \sin \omega_{i 0}\left(\tau-\tau_{i j}\right)}{2\left(1-\cos \omega_{i 0}\right)}+j\left(\frac{\sin \omega_{i 0}\left(\tau-\tau_{i j}\right)}{2}-\frac{\sin \omega_{i 0} \cos \omega_{i 0}(\tau-\tau i j)}{2\left(1-\cos \omega_{i 0}\right)}\right)\right\}\right| .
\end{aligned}
$$

After some manipulation, we get

$$
\begin{gathered}
a^{2}-a\left(\frac{\alpha d_{i}}{\beta}-2 \cdot \frac{\cos \omega_{i 0}(1+\tau)-\cos \omega_{i 0} \tau}{\beta}\right)+2 \\
\cdot \frac{1-\cos \omega_{i 0}}{\beta^{2}}-2 \cdot \frac{\alpha d_{i} \cos \omega_{i 0}(1+\tau)}{\beta^{2}}>0 .
\end{gathered}
$$

It is easy to see that (19) holds for $a>1$ if and only if the following two inequalities are satisfied:

$$
\begin{gathered}
2 \beta-\alpha d_{i}>4 \sin \frac{\omega_{i 0}}{2} \sin \frac{(1+2 \tau) \omega_{i 0}}{2}, \\
1-\frac{\alpha d_{i}}{\beta}+2 \cdot \frac{\cos \omega_{i 0}(1+\tau)-\cos \omega_{i 0} \tau}{\beta}+2 \\
\cdot \frac{1-\cos \omega_{i 0}}{\beta^{2}}-2 \cdot \frac{\alpha d_{i} \cos \omega_{i 0}(1+\tau)}{\beta^{2}}>0 .
\end{gathered}
$$

As $\beta>0$, we can obtain

$$
\begin{gathered}
\beta^{2}-\alpha \beta d_{i}+2 \beta\left[\cos \omega_{i 0}(1+\tau)-\cos \omega_{i 0} \tau\right]+2 \\
\cdot\left(1-\cos \omega_{i 0}\right)-2 \alpha d_{i} \cos \omega_{i 0}(1+\tau)>0
\end{gathered}
$$

The inequation (22) can be satisfied if the following two inequations hold,

$$
\begin{array}{r}
\beta^{2}-\alpha \beta d_{i}-2 \alpha d_{i} \cos \omega_{i 0}(1+\tau)>0, \\
\beta\left[\cos \omega_{i 0}(1+\tau)-\cos \omega_{i 0} \tau+1-\cos \omega_{i 0}>0 .\right.
\end{array}
$$

It is easy to know that $\cos \omega_{i 0}(1+\tau) \leq 1$ and from (23) we have $\beta^{2} /(\beta+2)>\alpha d_{i}$. According to (24) and based on Lemma 5 , we have $\tau<1 / 2 \beta-1 / 2$.

In addition, since $\tau>0$, we get

$$
\begin{aligned}
\frac{\beta^{2}}{\alpha(\beta+2)}>\max \left\{d_{i}\right\}, \\
\tau \in\left[0, \frac{1}{2 \beta}-\frac{1}{2}\right] .
\end{aligned}
$$

When $i \in \sigma_{2}$, the following inequality can also be obtained by Gerschgorin circle theorem:

$$
\begin{aligned}
G_{i} & =\left\{x: x \in|| x-\frac{\gamma}{e^{j \omega}-1} \sum_{j \in N_{i}} a_{i j} e^{-j \omega \tau} \mid\right. \\
& \left.\leq \sum_{j \in N_{i}}\left|\frac{\gamma a_{i j}}{e^{j \omega}-1} e^{-j \omega \tau_{i j}}\right|\right\} .
\end{aligned}
$$

Define $\widehat{d}_{i}=\sum_{j \in N_{i}} a_{i j}, i \in \sigma_{2}$. If the point $(-a, j 0), a \geq 1$ is not enclosed in $G_{i}, i \in \sigma_{2}$, it follows that

$$
\left|-a-\frac{\gamma \widehat{d}_{i}}{e^{j \omega}-1} e^{-j \omega \tau}\right|>\sum_{j \in N_{i}}\left|\frac{\gamma a_{i j}}{e^{j \omega}-1} e^{-j \omega \tau_{i j}}\right| .
$$

After some manipulations, we have

$$
a^{2}-a \gamma \widehat{d}_{i} \frac{\cos \omega \tau-\cos \omega(1+\tau)}{1-\cos \omega}>0 .
$$

From (28), we get

$$
\begin{aligned}
& \gamma<\frac{1}{\max \left\{\hat{d}_{i}\right\}}, \\
& \tau \in\left[0, \frac{1}{2 \gamma \max \left\{\hat{d}_{i}\right\}}-\frac{1}{2}\right] .
\end{aligned}
$$

Combining the analysis aforementioned, Theorem 6 is proved.

In Theorem 6, agents with identical input time delay and different communication time delay are investigated. Next, we will extend our work to the case of different input and communication delays. Based on (5) and (6), considering the following systems listed as (30) and (31):

$$
\begin{aligned}
x_{i}(k+1)= & x_{i}(k)+v_{i}(k), \\
v_{i}(k+1)= & v_{i}(k) \\
& -\alpha\left[\sum_{j \in N_{i}} a_{i j}\left(x_{j}\left(k-\tau_{i j}\right)+x_{i}\left(k-\tau_{i}\right)\right)\right] \\
& -\beta v_{i}(k-\tau), \quad i \in \sigma_{1}
\end{aligned}
$$

and

$$
\begin{array}{r}
x_{l}(k+1)=x_{l}(k) \\
-\gamma\left[\sum_{j \in N_{l}} a_{l j}\left(x_{j}\left(k-\tau_{l j}\right)+x_{l}\left(k-\tau_{l}\right)\right)\right], \\
l \in \sigma_{2},
\end{array}
$$

where $\tau_{i j}$ denotes the communication time delay from the $j$ th agent to the $i$ th agent, $\tau_{i}$ denotes the input time delay of the $i$ th agent. $\alpha, \beta, \gamma>0$ are the control parameters of the systems. 
Corollary 7. Couple-group consensus for the heterogeneous MASs (30) and (31) under bipartite digraph topology with a spanning tree can be achieved if the following conditions are satisfied:

(i) there exists a real number $\omega_{i 0} \geq 0$ satisfying the equation $\alpha d_{i}=4 \sin \left(\omega_{i 0} / 2\right) \cos \left((1+2 \tau) \omega_{i 0} / 2\right) \tan \left(\omega_{i 0} / 2\right)$ so that the inequality $2 \beta-\alpha d_{i}>4 \sin \left(\omega_{i 0} / 2\right) \sin ((1+$ $\left.2 \tau) \omega_{i 0} / 2\right)$ holds;

(ii) $\beta^{2} / \alpha(\beta+2)>\max \left\{d_{i}\right\}, \gamma<1 / \max \left\{\widehat{d}_{i}\right\}$;

(iii) $\tau_{i} \in[0,1 / 2 \beta-1 / 2], i \in \sigma_{1}$ and $\tau_{i} \in\left[0,1 / 2 \gamma \widehat{d}_{i}-\right.$ $1 / 2], i \in \sigma_{2}$, where $d_{i}=\sum_{j \in N_{i}} a_{i j}, i \in \sigma_{1}$ and $\hat{d}_{i}=$ $\sum_{j \in N_{i}} a_{i j}, i \in \sigma_{2}$, respectively.

Based on Lemmas 2 and 5, as the proof is similar to Theorem 6, we omit it here.

Remark 8. The results in Theorem 6 and Corollary 7 indicate that the control parameters of the system $\alpha, \beta, \gamma$ and the coupling weight of the agent interaction $d_{i}$ are critical factors for achieving group consensus. Furthermore, the reduction in either control parameters or coupling weights can make the system more tolerant of input time delays. However, communication time delays do not play a key role in achieving the group consensus.

Remark 9. Corollary 7 shows that the maximum input time delay that each node with respective dynamics can tolerate is different; it is decided by the control parameter of the system and the coupling weights impacted by its neighbors, which has the same dynamics. Similarly, the realization of the group consensus is not affected by the communication delays.

Remark 10. In order to achieve group consensus, many control protocols have been proposed generally based on the cooperative relationship among agents. According to the feature of the bipartite graph, that is, the information exchange of agents only occuring between different subgroups, we design protocols (3) and (4) based on the competitive relationship. This relaxes the condition that system topology needs to satisfy the in-degree balance when using cooperative protocols. And this also gives us a new perspective to investigate the group consensus problems.

\subsection{Group Consensus for Discrete-Time Heterogeneous MASs} with Multiple Time Delays under Digraph with In-Degree Balance. In order to meet the demand that information exchange of agents exists within the same subgroup, next, we will discuss the case of the heterogeneous MASs with multiple time delays under directed graph with in-degree balance.

Assumption 11. $\sum_{j \in N_{i}} a_{i j}=0, i \in L_{1}$ and $\sum_{j \in N_{l}} a_{l j}=0, l \in$ $L_{2}$, where $L_{1}$ and $L_{2}$ denote two subgroups.

Remark 12. As in $[3,5,18]$, Assumption 11 means that the interaction between the two subgroups is balanced, which is called the in-degree balance. This is necessary when group consensus is discussed in most situations.
Inspired by the control protocol based on the cooperative relationship, the system dynamic is designed and the closed form is listed as follows:

$$
\begin{aligned}
x_{i}(k+1)= & x_{i}(k)+v_{i}(k), \\
v_{i}(k+1)= & v_{i}(k) \\
& +\alpha\left[\sum_{v_{j} \in N_{I i}} a_{i j}\left(x_{j}\left(k-\tau_{i j}\right)-x_{i}(k-\tau)\right)\right. \\
& \left.+\sum_{v_{j} \in N_{I I i}} a_{i j} x_{j}\left(k-\tau_{i j}\right)\right]-\beta v_{i}(k-\tau), \\
& \quad i \in \sigma_{1}
\end{aligned}
$$

and

$$
\begin{aligned}
x_{i}(k+1)= & x_{i}(k)+v_{i}(k), \\
v_{i}(k+1)= & v_{i}(k) \\
& +\gamma\left[\sum_{v_{j} \in N_{I l}} a_{l j}\left(x_{j}\left(k-\tau_{l j}\right)-x_{l}(k-\tau)\right)\right. \\
& \left.+\sum_{v_{j} \in N_{I l}} a_{l j} x_{j}\left(k-\tau_{l j}\right)\right] .
\end{aligned}
$$

where $N_{I i}$ and $N_{I I i}$ are the neighbor sets of the agent $i$, which belong to the same and different subgroups of $i$, respectively.

Theorem 13. For the heterogeneous MASs (32) and (33) under directed graph topology with a spanning tree, the group consensus can be achieved if the following conditions are satisfied:

\section{(i) Assumption 11 holds;}

(ii) there exists a real number $\omega_{i 0} \geq 0$ satisfying the equation $\alpha d_{i}=4 \sin \left(\omega_{i 0} / 2\right) \cos \left((1+2 \tau) \omega_{i 0} / 2\right) \tan \left(\omega_{i 0} / 2\right)$ so that the inequality $2 \beta-\alpha d_{i}>4 \sin \left(\omega_{i 0} / 2\right) \sin ((1+$ $\left.2 \tau) \omega_{i 0} / 2\right)$ holds;

(iii) $\beta^{2} / \alpha(\beta+2)>\max \left\{d_{i}\right\}, \gamma<1 / \max \left\{\widehat{d}_{i}\right\}, \tau \in[0$, $\left.\min \left\{1 / 2 \beta-1 / 2,1 / 2 \gamma \max \left\{\widehat{d}_{i}\right\}-1 / 2\right\}\right]$, where $d_{i}=$ $\sum_{j \in N_{i}} a_{i j}, i \in \sigma_{1}$ and $\widehat{d}_{i}=\sum_{j \in N_{i}} a_{i j}, \quad i \in \sigma_{2}$.

Proof. Let the heterogeneous systems' dynamics (32) and (33) do $z$-transformation. Assuming $x_{i}(0)=0$, we have

$$
\begin{aligned}
(z-1)^{2} x_{s}(z)= & -\alpha\left(\widehat{L}_{s}+\widehat{D}_{s f}\right) x_{s}(z)+\alpha \widehat{A}_{s f} x_{f}(z) \\
& -(z-1) \beta e^{-\tau} x_{s}(z), \\
(z-1) x_{f}(z)= & \gamma \widehat{A}_{f_{s}} x_{s}(z)-\gamma\left(\widehat{L}_{f}+\widehat{D}_{f s}\right) x_{f}(z) .
\end{aligned}
$$

Define $x(z)=\left(x_{s}(z), x_{f}(z)\right)^{T}$, then (34) can be rewritten as

$$
(z-1) x(z)=\widehat{\Gamma}(z) x(z),
$$


where

$$
\begin{array}{lc}
\widehat{\Gamma}(z) & \left(\begin{array}{cc}
\frac{-(z-1)^{2} I-\alpha\left(\widehat{L}_{s}+\widehat{D}_{s f}\right)}{\beta z^{-\tau}} & \frac{\alpha \widehat{A}_{s f}}{\beta z^{-\tau}} \\
\gamma \widehat{A}_{f s} & -\gamma\left(\widehat{L}_{f}+\widehat{D}_{f s}\right)
\end{array}\right) .
\end{array}
$$

The characteristic equation of system (35) is

$$
\operatorname{det}((z-1) I-\widehat{\Gamma}(z))=0 .
$$

According to Lyapunov stability theory, the consensus of the system can be realized if the roots of (37) are either at $z=1$ or in the unit circle of the complex plane. Next, we discuss these two cases separately.

When $z=1, \operatorname{det}((z-1) I-\widehat{\Gamma}(z))=(\alpha / \beta)^{n}(-\gamma)^{m} \operatorname{det}(\widehat{L})$. Based on Lemma 3, we know that zero is a simple eigenvalue of $\widehat{L}$. Then the roots of (37) are at $z=1$.

When $z \neq 1$, define $\operatorname{det}((z-1) I-\widehat{\Gamma}(z))=\operatorname{det}(I+H(z))$, where $H(z)=-\widehat{\Gamma}(z) /(z-1), z=e^{j w}$. Based on general Nyquist criteria, one can know that the roots of (37) can be located in the unit circle of the complex plane if and only if the point $(-1, j 0)$ is not enclosed by the Nyquist curve of $H\left(e^{j w}\right)$. Hence, according to the Gerschgorin circle theorem, we have

$$
\lambda\left(H\left(e^{j \omega}\right)\right) \in\left\{H_{i}, i \in \sigma_{1}\right\} \cup\left\{H_{i}, i \in \sigma_{2}\right\} .
$$

When $i \in \sigma_{1}$, it yields that

$$
\begin{aligned}
& H_{i} \\
& \quad=\left\{x: x \in|| x-\frac{\alpha}{\left(e^{j \omega}-1\right) \beta} \sum_{j \in N_{i}} a_{i j}-\frac{e^{j \omega}-1}{\beta} e^{j \omega \tau} \mid\right. \\
& \left.\leq \sum_{j \in N_{i}}\left|\frac{\alpha a_{i j}}{\left(e^{j \omega}-1\right) \beta} e^{-j \omega\left(\tau_{i j}-\tau\right)}\right|\right\} .
\end{aligned}
$$

Noting that this inequation is similar to those in Theorem 6 , then after some derivation processes, we can obtain

$$
\tau \leq \frac{1}{2 \beta}-\frac{1}{2} .
$$

When $i \in \sigma_{2}$, the following inequality can also be obtained by the Gerschgorin circle theorem,

$$
\begin{aligned}
H_{i} & =\left\{x: x \in\left|x-\frac{\gamma}{e^{j \omega}-1} \sum_{j \in N_{i}} a_{i j} e^{-j \omega \tau}\right|\right. \\
& \left.\leq \sum_{j \in N_{i}}\left|\frac{\gamma a_{i j}}{e^{j \omega}-1} e^{-j \omega \tau_{i j}}\right|\right\} .
\end{aligned}
$$

Then we can get

$$
\tau \leq \frac{1}{2 \gamma \widehat{d}_{i}}-\frac{1}{2} .
$$

Combining the above analysis, this finishes the proof of Theorem 13.
Based on (32) and (33), consider the following dynamics as (43) and (44):

$$
\begin{aligned}
x_{i}(k+1)= & x_{i}(k)+v_{i}(k), \\
v_{i}(k+1)= & v_{i}(k) \\
& +\alpha\left[\sum_{v_{j} \in N_{I i}} a_{i j}\left(x_{j}\left(k-\tau_{i j}\right)-x_{i}\left(k-\tau_{i}\right)\right)\right. \\
& \left.+\sum_{v_{j} \in N_{I I}} a_{i j} x_{j}\left(k-\tau_{i j}\right)\right] \\
& -\beta v_{i}\left(k-\tau_{i}\right), \quad i \in \sigma_{1}
\end{aligned}
$$

and

$$
\begin{aligned}
x_{i}(k+1)= & x_{i}(k)+v_{i}(k), \\
v_{i}(k+1)= & v_{i}(k) \\
& +\gamma\left[\sum_{v_{j} \in N_{I l}} a_{l j}\left(x_{j}\left(k-\tau_{l j}\right)-x_{l}(k-\tau)\right)\right. \\
& \left.+\sum_{v_{j} \in N_{I l}} a_{l j} x_{j}\left(k-\tau_{l j}\right)\right], \quad i \in \sigma_{2} .
\end{aligned}
$$

Corollary 14. For the heterogeneous MASs (43) and (44) under directed graph topology with a spanning tree, the group consensus can be achieved if the following conditions are satisfied:

(i) Assumption 11 holds;

(ii) there exists a real number $\omega_{i 0} \geq 0$ satisfying the equation $\alpha d_{i}=4 \sin \left(\omega_{i 0} / 2\right) \cos \left((1+2 \tau) \omega_{i 0} / 2\right) \tan \left(\omega_{i 0} / 2\right)$ so that the inequality $2 \beta-\alpha d_{i}>4 \sin \left(\omega_{i 0} / 2\right) \sin ((1+$ $\left.2 \tau) \omega_{i 0} / 2\right)$ holds;

(iii) $\beta^{2} / \alpha(\beta+2)>\max \left\{d_{i}\right\}, \gamma<1 / \max \left\{\widehat{d}_{i}\right\}$;

(iv) $\tau_{i} \in\left[0, \beta^{2} / 2 \beta-1 / 2\right], i \in \sigma_{1}$ and $\tau_{i} \in\left[0,1 / 2 \gamma \widehat{d}_{i}-\right.$ $1 / 2], i \in \sigma_{2}$, where $d_{i}=\sum_{j \in N_{i}} a_{i j}, i \in \sigma_{1}$ and $\widehat{d}_{i}=$ $\sum_{j \in N_{i}} a_{i j}, i \in \sigma_{2}$, respectively.

We omit the proof of Corollary 14 as it is similar to Theorem 13.

Remark 15. The dynamics of the agents in our work do not contain the virtual velocity estimation, which exists in many related works $[15,17,19]$ for the sake of analyzing heterogeneous systems. In those works, with the view of imitating the dynamics of the second-order agents, the velocity estimation is added to the first-order agents, but this requires extra computation and wastes system's resources.

Remark 16. In this paper, the topology of the systems we considered is either a bipartite digraph or a digraph satisfying in-degree balance, both of them has a spanning tree. They 


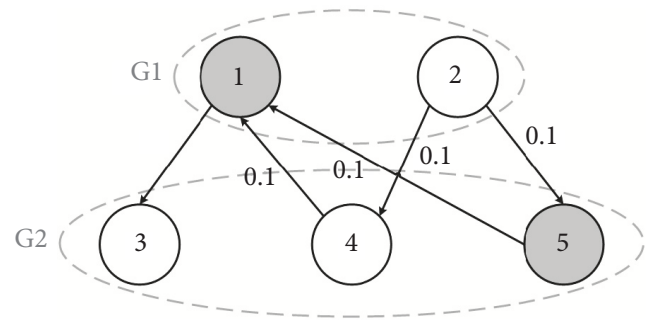

FIGURE 1: The bipartite digraph topology of the heterogeneous MASs.

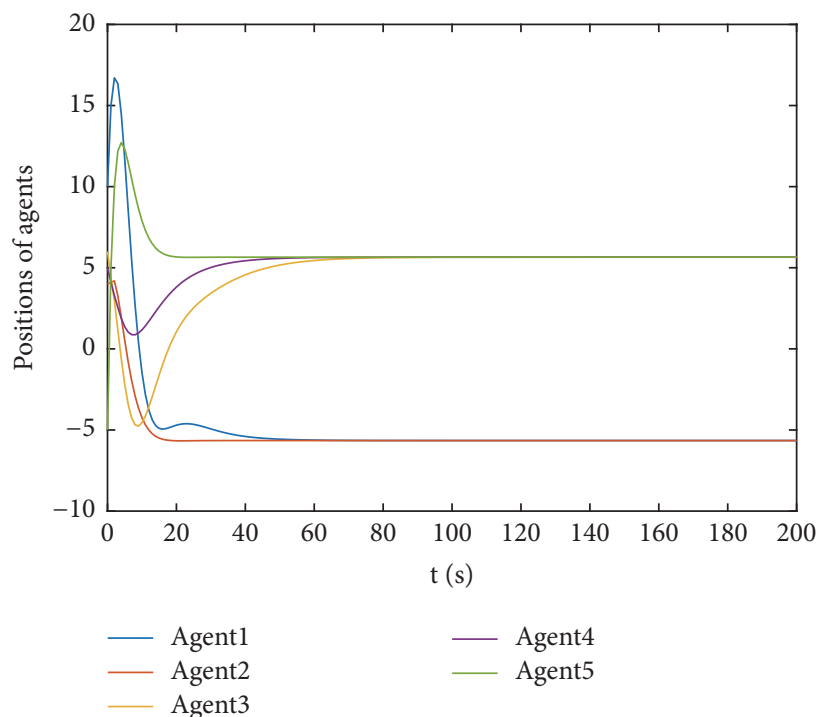

(a)

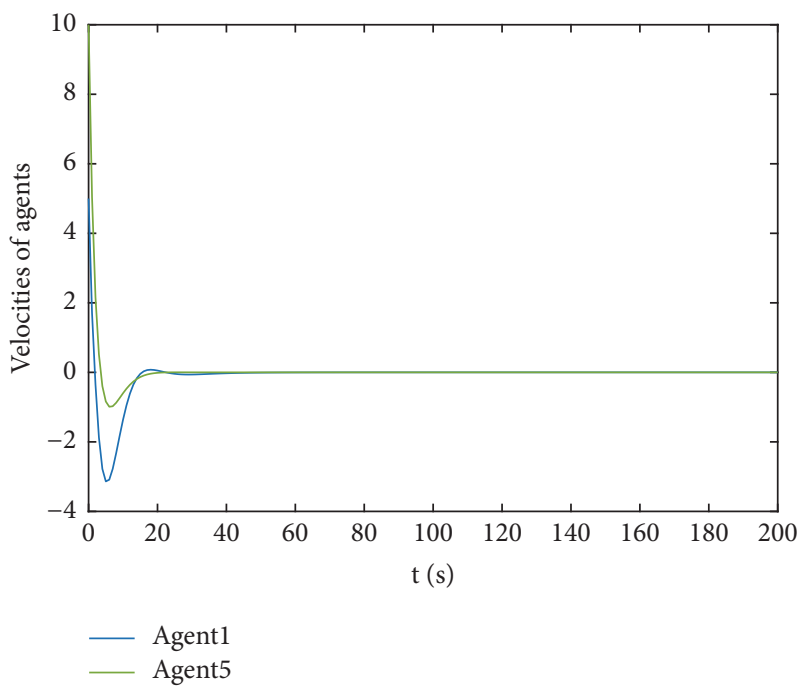

(b)

FIgURE 2: The state trajectories of the agents under directed topology in Figure 1 with identical input time delays $\tau=0.5, \tau_{i j}=1$. (a) Positions, (b) Velocities.

seem to be more specific. But in fact, group consensus for heterogeneous systems usually can hardly be achieved unless adding some stronger conditions. As in [15-19], the systems' topology is undirected or containing a spanning tree; meanwhile, in those papers in order to achieve the group consensus, additional assumptions are specified, like in-degree balance and the geometric multiplicity of zero eigenvalues of the system's Laplacian matrix which are at least two. It will be a challenge to discuss the problem of the group consensus for heterogeneous MASs under a more general condition.

Remark 17. Note that all the results in this paper are theoretically obtained in the case $x_{i}(k), v_{i}(k), u_{i}(k) \in \mathbb{R}$. Utilizing the Kronecker product, they can be generalized to $x_{i}(k), v_{i}(k)$, $u_{i}(k) \in \mathbb{R}^{n}, n>1$.

\section{Simulation}

In this subsection, the effectiveness of the obtained results is demonstrated by several simulations.

Example 18. Figure 1 shows a heterogeneous system with the bipartite digraph topology which has a spanning tree. As known, the whole system is divided into two subgroups: $G_{1}$ and $G_{2}$. Hereinto, agents 1 and 2 and 3, 4, and 5 belong to these two subgroups. Without loss of generality, we further assume that agents 1 and 5 are second-order and that the dynamics of the remaining agents are first-order. In our scenario, the dynamics of agents in the same subgroup do not need to be the same, which is different from the case in [16-19]. Obviously, those are special cases of ours.

For simplicity, we assume each edge's weight as $a_{i j}=$ $0.1, i, j \in[1,5]$, and it is easy to obtain the in-degree of each agent: $d_{1}=0.2, d_{2}=0.1, d_{3}=0.1, d_{4}=0.1$, and $d_{5}=0.1$. Based on Theorem 6 , we set $\alpha=0.4$, $\beta=0.5$, and $\gamma=1$, then the upper bound of input time delays is obtained, $\tau \in[0, \min \{1 / 2,9 / 2\}]$. In the simulation, we choose $\tau=0.5$ and all the conditions in Theorem 6 are satisfied now. Since the realization of the group consensus is independent from the communication time delays, for convenience, we choose their values to be the same as $\tau_{i j}=1$. The trajectories of the agents of the systems (5) and (6) are shown in Figure 2. The results indicate that the systems' couple-group consensus is reached asymptotically. 


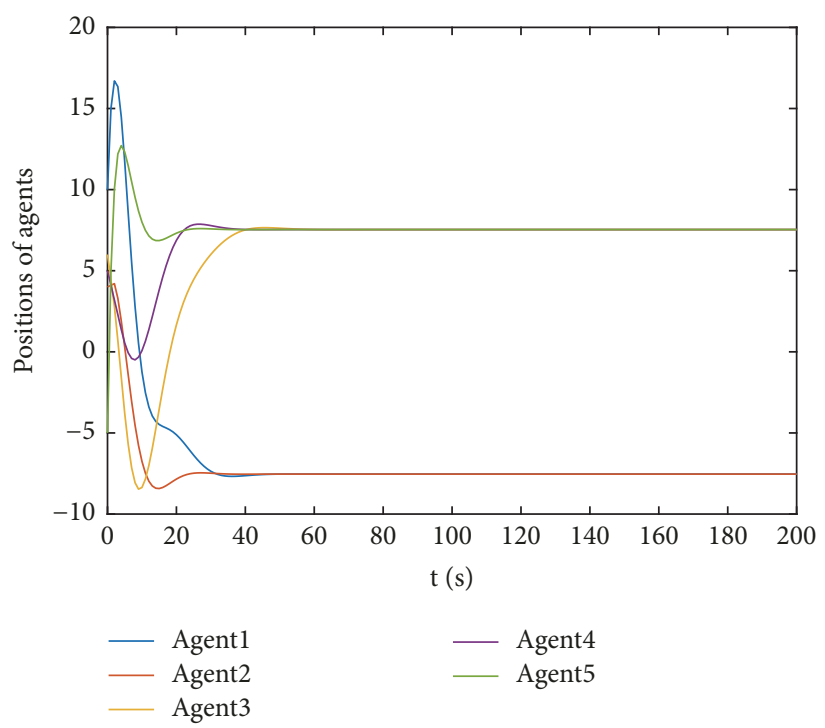

(a)

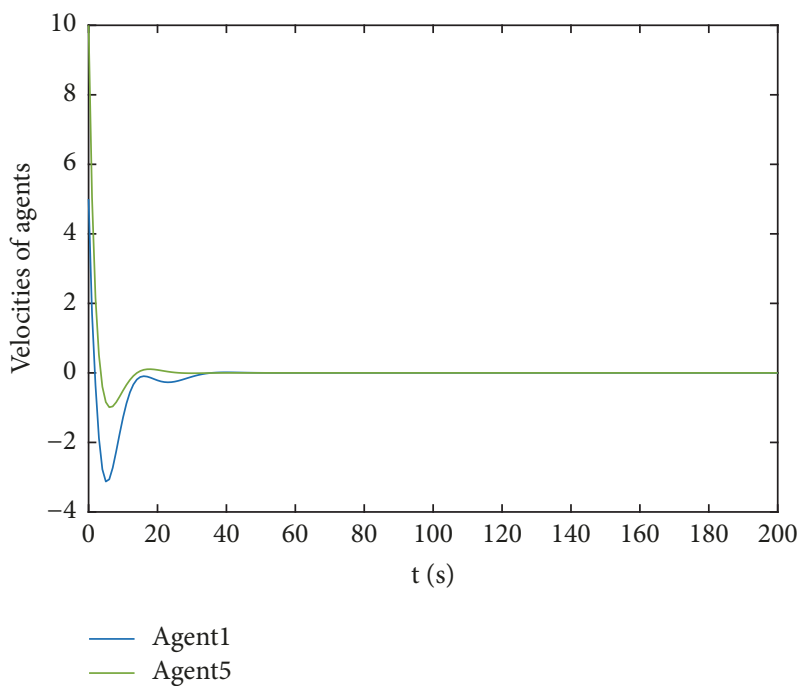

(b)

FIgURE 3: The state trajectories of the agents under directed topology in Figure 1 with different input time delays $\tau_{1}=0.5, \tau_{2}=4.5, \tau_{3}=4.5$, $\tau_{4}=4.5, \tau_{5}=0.5$, and $\tau_{i j}=1$. (a) Positions, (b) Velocities.

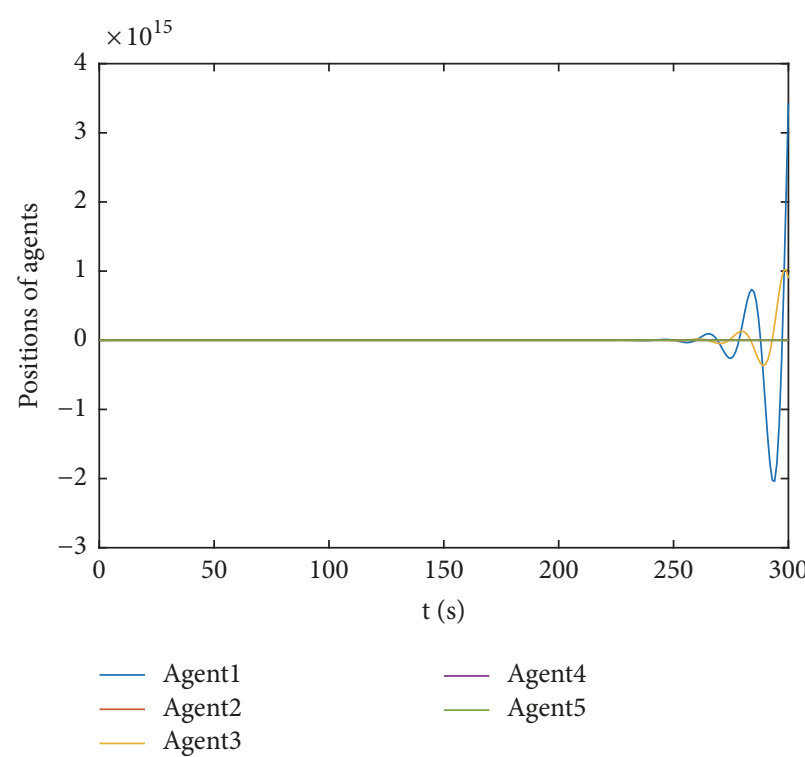

(a)

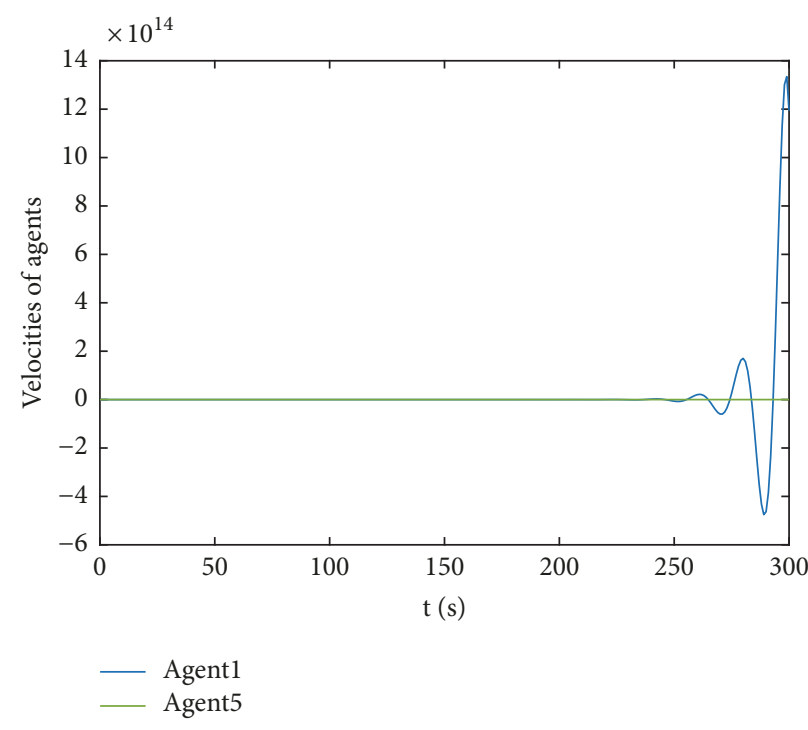

(b)

FIgURE 4: The state trajectories of the agents under directed topology in Figure 1 with different input time delays $\tau_{1}=4.5, \tau_{2}=4.5, \tau_{3}=4.5$, $\tau_{4}=4.5, \tau_{5}=0.5$, and $\tau_{i j}=1$. (a) Positions, (b) Velocities.

From Corollary 7 and according to the control parameters given above, the upper bound of input delays can be calculated for each node: $\tau_{1} \leq 1 / 2, \tau_{2} \leq 9 / 2, \tau_{3} \leq 9 / 2, \tau_{4} \leq 9 / 2$, and $\tau_{5} \leq 1 / 2$. In this case, we choose $\tau_{1}=0.5, \tau_{2}=4.5$, $\tau_{3}=4.5, \tau_{4}=4.5, \tau_{5}=0.5$. It is clear that the conditions in Corollary 7 are all satisfied here. Figure 3 demonstrates that the couple-group consensus is realized. Furthermore, if we set one of the input delays, $\tau_{1}=4.5$ for instance, to violate the upper bound we proposed, from the trajectory in Figure 4, we know that the system is divergent.
Next, we will testify Theorem 13 and Corollary 14.

Example 19. Figure 5 shows an in-degree balance graph and $d_{1}=0.1, d_{2}=0.1, d_{3}=0.1, d_{4}=0.1$, and $d_{5}=0.1$. Set $\alpha=0.2, \beta=0.3, \gamma=1$, and $\tau=1$. Obviously, the conditions of Theorem 13 are satisfied. The trajectories of the agents are shown in Figure 6. Consider the systems (34) and (35), and choose different input time delays as $\tau_{1}=1, \tau_{2}=4.5$, $\tau_{3}=4.5, \tau_{4}=4.5$, and $\tau_{5}=1$. Here, Corollary 14 is satisfied, and the trajectories are shown in Figure 7. From Figures 6 


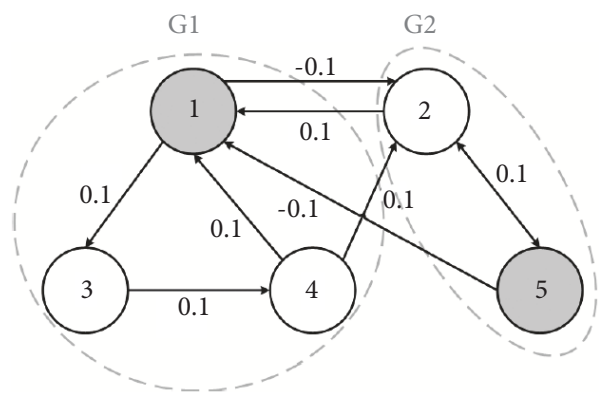

FIGURE 5: The directed graph topology of the heterogeneous MASs.

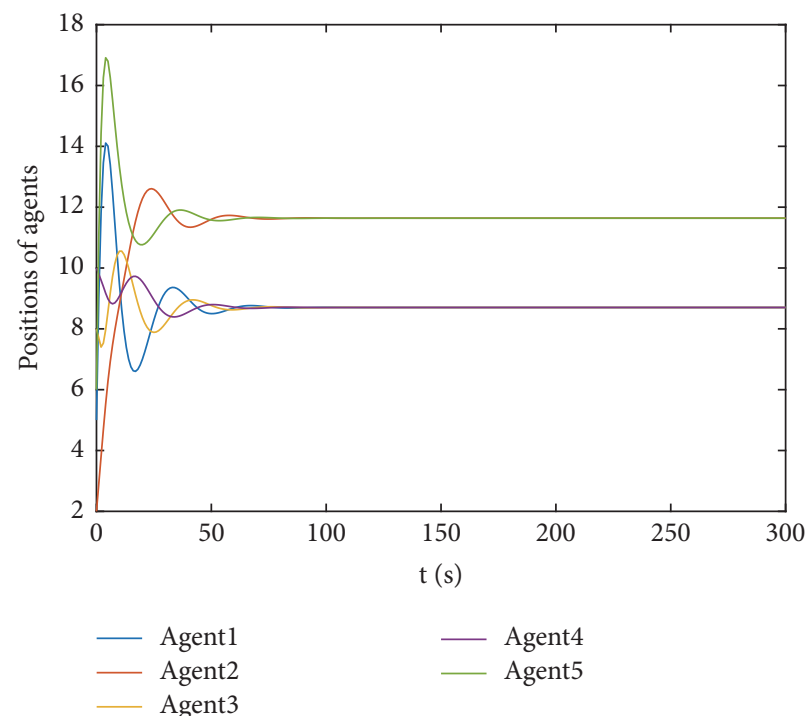

(a)

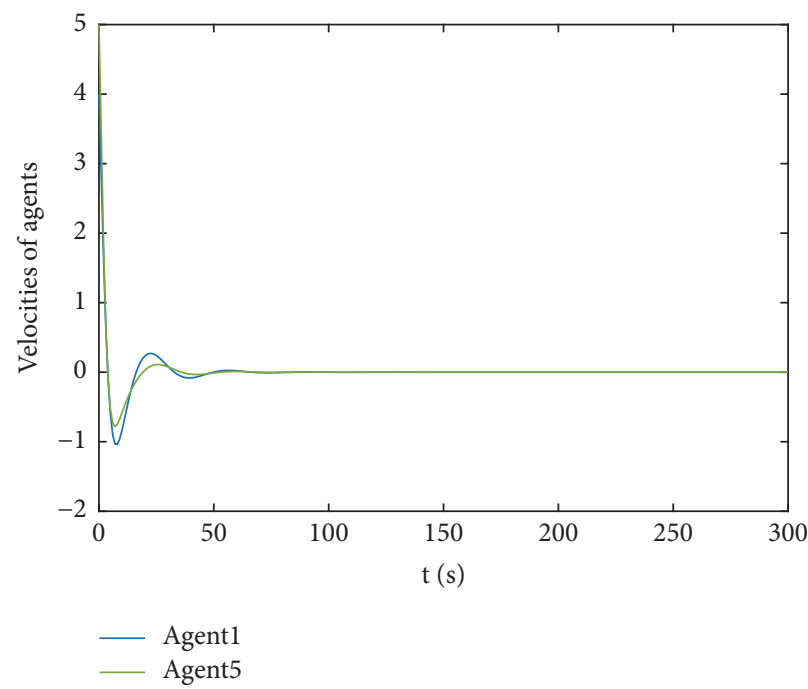

(b)

Figure 6: The state trajectories of the agents under directed topology in Figure 5 with identical input time delays $\tau=1$, $\tau_{i j}=1$. (a) Positions, (b) Velocities.

and 7 we know that the couple-group consensus is achieved asymptotically. When set $\tau_{5}=4.5$ exceeds the upper bound, the system diverges (Figure 8).

Remark 20. In this paper, we designed a competition-based control protocol under bipartite graph and a cooperationbased protocol under in-degree balance graph to realize group consensus. Competitive protocol will lead to a couple of agents which belong to two different subgroups apart from each other. In this case, agents in the same subgroup have no interactions, and the final states of the system are only two and opposite, which is called the couple-group consensus. Cooperative protocol will lead the agents in one subgroup to be close to each other, while the different subgroups need to satisfy the in-degree balance, which means all agents from one subgroup impose a weight of zero on any node within another subgroup. In this case, more than two system states can be achieved. Both approaches have their own limitation of topology. Next, we plan to combine the competition and cooperation ideas to study the group consensus problem under more general system topology.

\section{Conclusion}

Under two forms of typical topologies, the couple-group consensus for the discrete-time heterogeneous MASs with input and communication time delays is investigated. For the bipartite graph, group consensus algorithms based on the competitive relationship between agents are designed; for the graph satisfying in-degree balance, we propose the algorithms based on cooperative relationship accordingly. Utilizing matrix theory and general Nyquist criteria, we theoretically propose some sufficient algebraic criteria and the upper bound of the input time delays for ensuring the consensus. From them, we find that the achievement of the couple-group consensus for the heterogeneous systems depends on the input time delays of agents, the control parameters of the systems, and the coupling weights between the agents, but it is independent of the communication time delays. The simulations verify the correctness of our theoretical results. Our future work will extend to more complicated group consensus problems of the heterogeneous multiagent systems, for example, considering sampled information or switching topology which commonly exist in reality. 


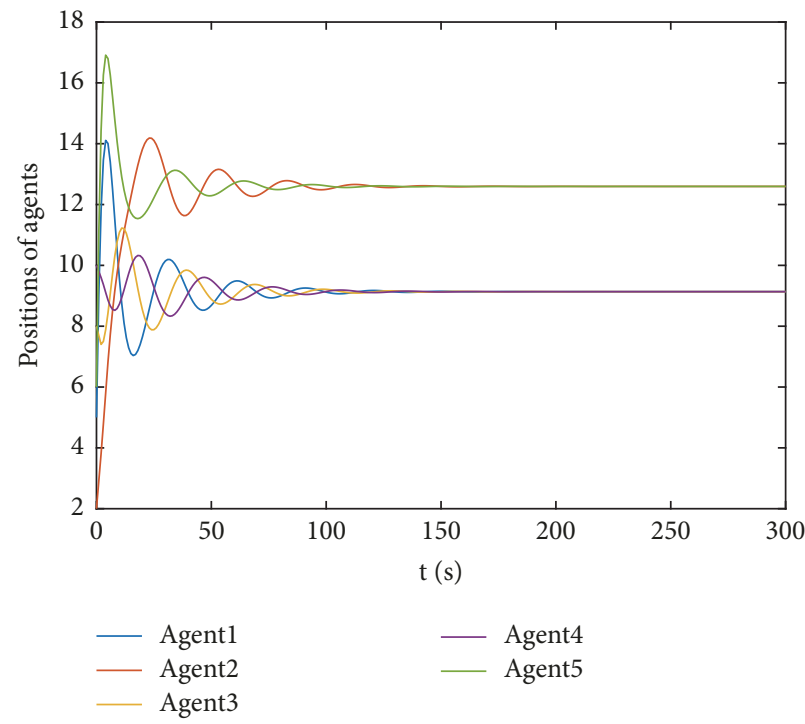

(a)

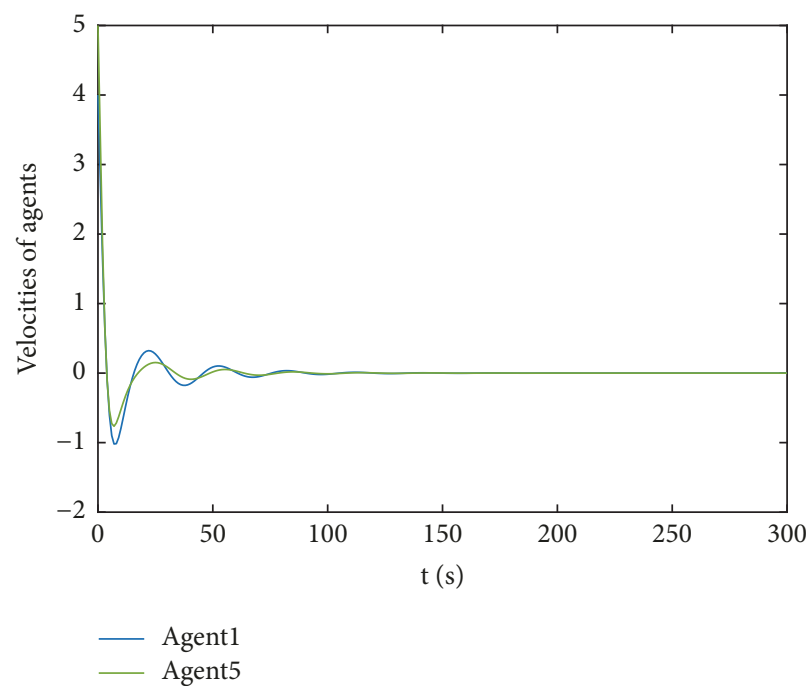

(b)

Figure 7: The state trajectories of the agents under directed topology in Figure 5 with different input time delays $\tau_{1}=1, \tau_{2}=4.5, \tau_{3}=4.5$, $\tau_{4}=4.5, \tau_{5}=1$, and $\tau_{i j}=1$. (a) Positions, (b) Velocities.

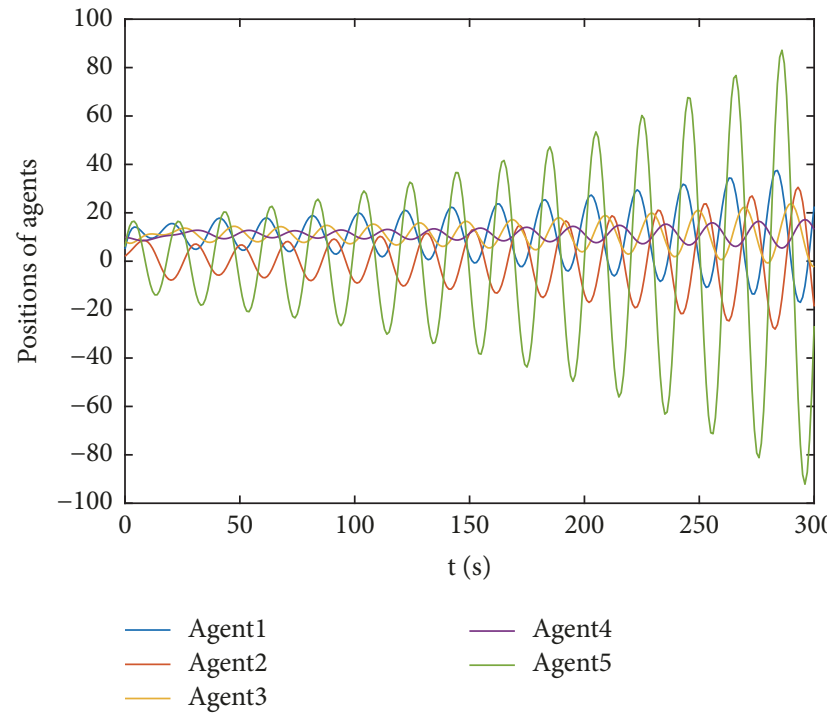

(a)

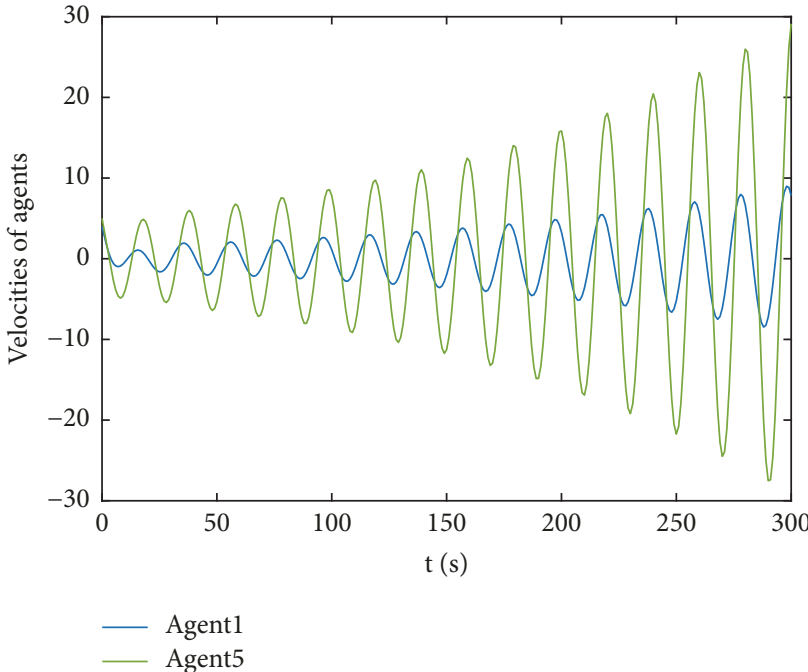

(b)

FIGURE 8: The state trajectories of the agents under directed topology in Figure 5 with different input time delays $\tau_{1}=1, \tau_{2}=4.5, \tau_{3}=4.5$, $\tau_{4}=4.5, \tau_{5}=4.5$, and $\tau_{i j}=1$. (a) Positions, (b) Velocities.

\section{Data Availability}

The data used to support the findings of this study are available from the corresponding author upon request.

\section{Conflicts of Interest}

The authors declare that they have no conflicts of interest.

\section{Acknowledgments}

This work was supported in part by the National Natural Science Foundation of China under Grant no. 61876200, in part by the Postgraduate Scientific Research and Innovation Project of Chongqing under Grant no. CYS18246, in part by the Natural Science Foundation Project of Chongqing Science and Technology Commission under Grant no. cstc2018jcyjAX0112, and in part by the Key Theme Special Project of Chongqing Science and Technology Commission under Grant nos. cstc2017zdcy-zdyfx0091 and cstc2017rgznzdyfx0022.

\section{References}

[1] Y. Zheng, J. Ma, and L. Wang, "Consensus of hybrid multi-agent systems," IEEE Transactions on Neural Networks and Learning Systems, vol. 29, no. 4, pp. 1359-1365, 2018. 
[2] Y. Zhu, S. Li, J. Ma, and Y. Zheng, "Bipartite consensus in networks of agents with antagonistic interactions and quantization," IEEE Transactions on Circuits and Systems II: Express Briefs, 2018.

[3] D. Xie and T. Liang, "Second-order group consensus for multiagent systems with time delays," Neurocomputing, vol. 153, pp. 133-139, 2015.

[4] H. Xia, T.-Z. Huang, J.-L. Shao, and J.-Y. Yu, "Group consensus of multi-agent systems with communication delays," Neurocomputing, vol. 171, pp. 1666-1673, 2016.

[5] Q. Cui, D. Xie, and F. Jiang, "Group consensus tracking control of second-order multi-agent systems with directed fixed topology," Neurocomputing, vol. 218, pp. 286-295, 2016.

[6] K. Liu, Z. Ji, G. Xie, and L. Wang, "Consensus for heterogeneous multi-agent systems under fixed and switching topologies," Journal of The Franklin Institute, vol. 352, no. 9, pp. 3670-3683, 2015.

[7] J. Sun and Z. Geng, "Adaptive consensus tracking for linear multi-agent systems with heterogeneous unknown nonlinear dynamics," International Journal of Robust and Nonlinear Control, vol. 26, no. 1, pp. 154-173, 2016.

[8] Y. Jiang, L. Ji, Q. Liu, S. Yang, and X. Liao, "Couple-group consensus for discrete-time heterogeneous multiagent systems with cooperative-competitive interactions and time delays," Neurocomputing, vol. 319, pp. 92-101, 2018.

[9] Y.-J. Sun, G.-L. Zhang, S.-X. Zhang, and J. Zeng, "Consensus equilibrium point analysis for a class of discrete-time heterogeneous multi-agent systems," Kongzhi yu Juece/Control and Decision, vol. 30, no. 8, pp. 1479-1484, 2015.

[10] C. Liu and F. Liu, "Stationary consensus of heterogeneous multi-agent systems with bounded communication delays," Automatica, vol. 47, no. 9, pp. 2130-2133, 2011.

[11] M. Di Bernardo, A. Salvi, and S. Santini, "Distributed consensus strategy for platooning of vehicles in the presence of timevarying heterogeneous communication delays," IEEE Transactions on Intelligent Transportation Systems, vol. 16, no. 1, pp. 102112, 2015.

[12] J. Yu and Y. Shi, "Scaled group consensus in multiagent systems with first/second-order continuous dynamics," IEEE Transactions on Cybernetics, 2017.

[13] Y. Feng, S. Xu, and B. Zhang, "Group consensus control for double-integrator dynamic multiagent systems with fixed communication topology," International Journal of Robust and Nonlinear Control, vol. 24, no. 3, pp. 532-547, 2014.

[14] Y. Shang, "Group consensus of multi-agent systems in directed networks with noises and time delays," International Journal of Systems Science, vol. 46, no. 14, pp. 2481-2492, 2015.

[15] C.-L. Liu and F. Liu, "Dynamical consensus seeking of heterogeneous multi-agent systems under input delays," International Journal of Communication Systems, vol. 26, no. 10, pp. 1243-1258, 2013.

[16] C. Liu, Q. Zhou, and X. Hu, "Group consensus of heterogeneous multi-agent systems with fixed topologies," International Journal of Intelligent Computing and Cybernetics, vol. 8, no. 4, pp. 294-311, 2015.

[17] G. Wen, J. Huang, C. Wang, Z. Chen, and Z. Peng, "Group consensus control for heterogeneous multi-agent systems with fixed and switching topologies," International Journal of Control, vol. 89, no. 2, pp. 259-269, 2016.

[18] G. Wen, Y. Yu, Z. Peng, and H. Wang, "Dynamical group consensus of heterogenous multi-agent systems with input time delays," Neurocomputing, vol. 175, pp. 278-286, 2015.
[19] Y. Zheng and L. Wang, "A novel group consensus protocol for heterogeneous multi-agent systems," International Journal of Control, vol. 88, no. 11, pp. 2347-2353, 2015.

[20] L. Wang and F. Xiao, "A new approach to consensus problems in discrete-time multiagent systems with time-delays," Science China Information Sciences, vol. 50, no. 4, pp. 625-635, 2007.

[21] Q. Wang and Y. Wang, "Cluster synchronization of a class of multi-agent systems with a bipartite graph topology," Science China Information Sciences, vol. 57, no. 1, pp. 1-11, 2014.

[22] Y. Tian and C. Liu, "Consensus of multi-agent systems with diverse input and communication delays," IEEE Transactions on Automatic Control, vol. 53, no. 9, pp. 2122-2128, 2008. 


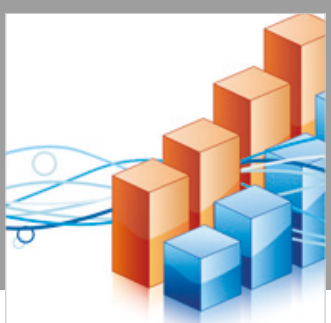

Advances in

Operations Research

\section{-n-m}
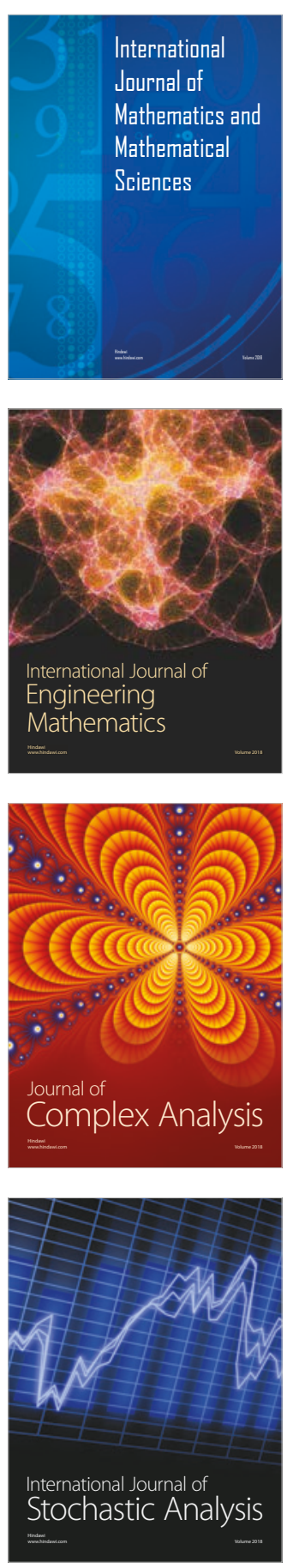
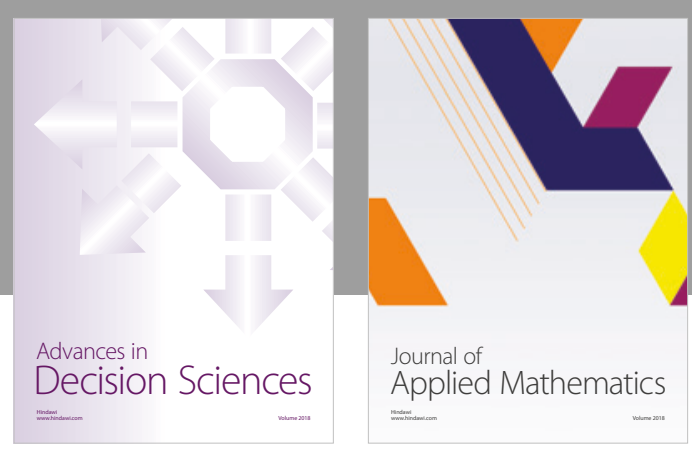

Journal of

Applied Mathematics
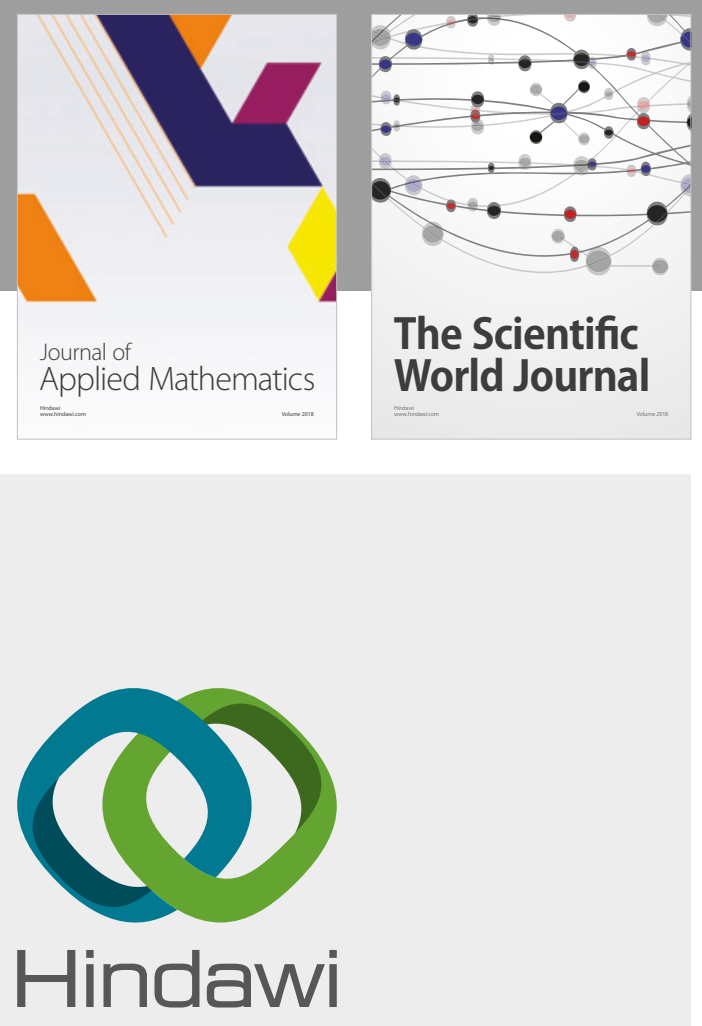

Submit your manuscripts at

www.hindawi.com

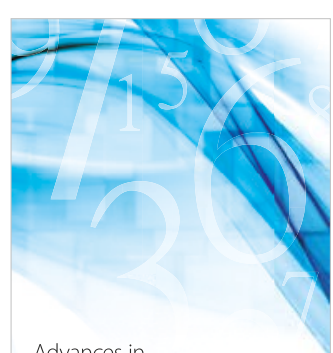

Advances in
Numerical Analysis
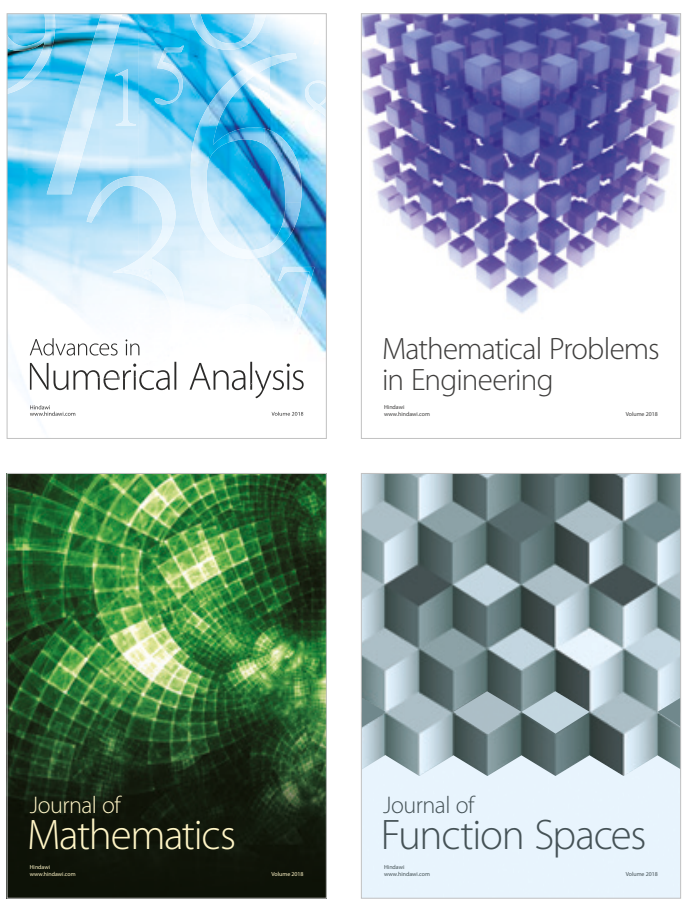

Mathematical Problems in Engineering

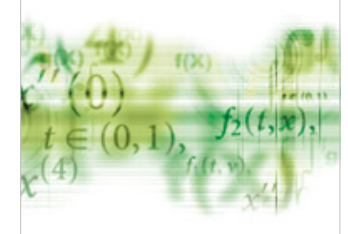

International Journal of

Differential Equations

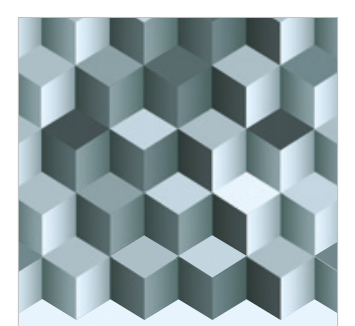

Journal of

Function Spaces
The Scientific

World Journal

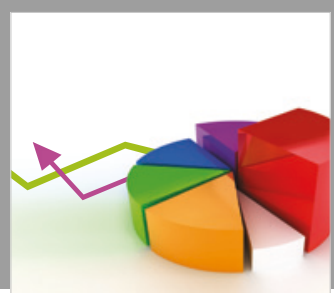

Journal of

Probability and Statistics
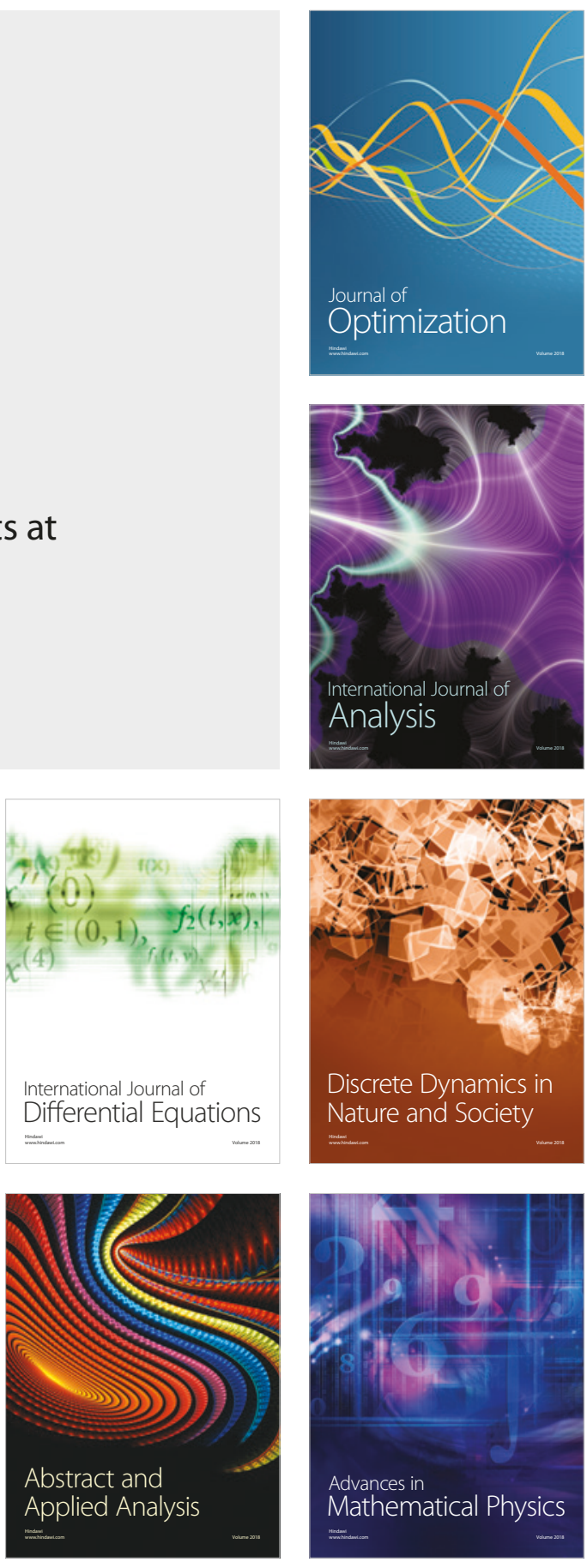